\title{
Use of walking modifications, perceived walking difficulty and changes in outdoor mobility among community-dwelling older people during COVID-19 restrictions
}

\author{
Heidi Leppä ${ }^{1}$ (D) $\cdot$ Laura Karavirta $^{1} \cdot$ Timo Rantalainen $^{1} \cdot$ Merja Rantakokko $^{2} \cdot$ Sini Siltanen $^{3} \cdot$ Erja Portegijs $^{1}$. \\ Taina Rantanen ${ }^{1}$
}

Received: 18 May 2021 / Accepted: 4 August 2021 / Published online: 20 August 2021

(c) The Author(s) 2021

\begin{abstract}
Background Outdoor mobility enables participation in essential out-of-home activities in old age.

Aim To compare changes in different aspects of outdoor mobility during COVID-19 restrictions versus two years before according to self-reported walking.

Methods Community-dwelling participants of AGNES study (2017-2018, initial age 75-85) responded to AGNESCOVID-19 postal survey in spring $2020(N=809)$. Life-space mobility, autonomy in participation outdoors, and self-reported physical activity were assessed at both time points and differences according to self-reported walking modifications and difficulty vs. intact walking at baseline were analyzed.

Results Life-space mobility and autonomy in participation outdoors had declined (mean changes -11.4, SD 21.3; and 6.7, SD 5.3, respectively), whereas physical activity had increased (5.5 $\mathrm{min} /$ day, SD 25.1) at follow-up. Participants perceiving walking difficulty reported the poorest baseline outdoor mobility, a steeper decline in life-space mobility $(p=0.001)$, a smaller increase in physical activity $(p<0.001)$, and a smaller decline in autonomy in participation outdoors $(p=0.017)$ than those with intact walking. Those with walking modifications also reported lower baseline life-space mobility and physical activity, a steeper decline in life-space mobility and a smaller increase in physical activity those with intact walking $(p<0.001$ for both). Discussion Participants reporting walking modifications remained the intermediate group in outdoor mobility over time, whereas those with walking difficulty showed the steepest decline in outdoor mobility and hence potential risk for accelerated further functional decline.

Conclusion Interventions should target older people perceiving walking difficulty, as they may be at the risk for becoming homebound when environmental facilitators for outdoor mobility are removed.
\end{abstract}

Keywords Aging $\cdot$ Compensation $\cdot$ Mobility $\cdot$ Participation $\cdot$ Social isolation $\cdot$ SARS-CoV-2

\section{Introduction}

Heidi Leppä

heidi.e.leppa@jyu.fi

1 Gerontology Research Center and Faculty of Sport and Health Sciences, University of Jyväskylä, Jyväskylä, Finland

2 JAMK University of Applied Sciences, Institute of Rehabilitation, Jyväskylä, Finland

3 Finnish Institute for Health and Welfare, Helsinki, Finland
Outdoor mobility indicates an individual's actual mobility behavior and perceived possibilities for participation in essential out-of-home activities [1,2]. The concept includes all types of journeys outside home, whether on foot or by other means of transportation, and thus requires some level of walking ability [2]. During the aging process, age-related diseases and functional decline may increase the risk for walking difficulty [3], in turn hindering possibilities to participate in out-of-home activities and leading to further decline in outdoor mobility [4]. However, before perceiving actual walking difficulty, older people noticing the first signs of functional decline may 
seek to maintain their outdoor mobility by modifying their walking behavior, for example, using an aid or walking more slowly [4].

During spring 2020, multiple actions were taken globally to slow down the spread of the SARS-CoV-2 virus responsible for COVID-19, especially among high-risk populations. In Finland, the government announced a state of emergency and, as a general guideline, advised older people to limit their physical contacts and avoid crowded areas. Restaurants, libraries, and indoor sport facilities were closed, and many cultural and civic society events and organized classes were canceled. Particular concerns were expressed regarding the potentially adverse consequences of these restrictions on older people's outdoor mobility and physical activity, as older people typically accumulate most of their physical activity while running daily errands, attending various events or making social visits [5-7].

Thus far, studies evaluating the effects of the COVID19 restrictions and lockdowns have focused on changes in one aspect of outdoor mobility at a time among older people and have mostly utilized cross-sectional data based on convenience samples [8-11]. In these studies, the majority of older people reported a decrease in their physical activity during the COVID-19 restrictions [8-10]. Lower scores for life-space mobility, referring to individuals' actual mobility behavior in daily life, and for active aging were observed in our previous study comparing data collected during the COVID-19 restrictions with data collected two years earlier [11]. In our previous prospective study [4] conducted prior to the COVID-19 pandemic, perceived walking difficulty preceded the decline in life-space mobility. However, the use of walking modifications enabled older people to postpone the decline in life-space mobility and in autonomy in participation outdoors [12]. It is thus possible that the COVID-19 restrictions have had different effects on older people's life-space mobility, autonomy in participation outdoors and physical activity, according to their use of walking modifications or perceived walking difficulty. We hypothesized that older people who perceived walking difficulty prior to the COVID-19 pandemic would show a steeper decline in various aspects of their outdoor mobility during the COVID-19 restrictions compared to those with intact walking.

The first aim of this study was to examine levels and changes in life-space mobility, autonomy in participation outdoors, and self-reported physical activity among older people during the COVID-19 restrictions compared to two years earlier. The second aim was to investigate whether the levels and changes in these various aspects of outdoor mobility differed between those reporting intact walking, walking modifications, or difficulty in walking a 2-km (km) distance at baseline.

\section{Methods}

\section{Study design and participants}

This study presents longitudinal results of the 'Active Aging - resilience and external support as modifiers of the disablement outcome' (AGNES) observational cohort study. Follow-up data (AGNES-COVID-19) were collected via postal questionnaires during the COVID-19 restrictions (May and June 2020) and these data were compared to baseline data (collected 2017-2018). The study protocol of the AGNES study [13] and non-respondent analyses of both datasets have been reported previously $[11,14]$. Briefly, the AGNES study is an observational study of three birth cohorts (aged 75, 80 and 85 years). A random sample based on age and residence in specific postal code areas in Jyväskylä (Finland) was drawn from the Digital and Population Data Services Agency in Finland. Inclusion criteria were being resident in the study area, community-dwelling, willing to participate, able to communicate, and provide an informed consent. At baseline, structured personal interview was conducted in participants homes $(\mathrm{N}=1018)$. At follow-up, a postal questionnaire was sent to the 985 baseline participants not known to have died or been transferred to an institutional care facility, and who had not withdrawn their consent [11]. Altogether, 809 responses were received. Seven participants had difficulty answering the questionnaire or preferred an interview and were thus interviewed over the phone. During collecting the follow-up data, the number of confirmed COVID-19 cases was low in the study area (102 cases, population 253 000, 21 municipalities) [11].

\section{Measurements}

Self-reported walking modifications and difficulty in 2-km were assessed at baseline $[15,16]$. First, perceived difficulty in walking a distance of 2-km was asked with the question: "Do you have difficulty in walking 2-km?" The response alternatives varied from "able to manage without difficulty" to "unable to manage even with help". Second, those using walking modifications at baseline were identified by asking those who reported being able to walk without difficulty an additional question: "Have you noticed any of the following changes due to your health or physical functioning when walking 2-km?" The response options were walking slower, taking rest breaks during walking, using an aid, having reduced the frequency of walking, and having given up walking distances of 2-km ("yes" or "no"). For the analyses, participants were categorized as follows: 1) intact walking (no difficulty nor modifications, 
reference), 2) walking modifications (no difficulty and at least one modification), and 3) walking difficulty (at least some difficulty).

Life-space mobility, autonomy in participation outdoors and self-reported physical activity were measured at baseline and during the COVID-19 restrictions. Life-space mobility was measured with the Finnish version of the University of Alabama at Birmingham Study of Aging Life-Space Assessment $[17,18]$. The Life-Space Mobility Assessment is a validated measure designed to capture individuals' actual mobility behavior in daily life. Participants were asked on how many days per week during the four weeks preceding the assessment they reached each life-space level, and if they needed help from other people or assistive devices. A higher life-space composite score indicates greater life-space mobility (range 0-120) [17].

Autonomy in participation outdoors was measured using the respective subscale of the Impact on Participation and Autonomy Questionnaire (IPA) [19]. The IPA is a validated measure for assessing participation and autonomy in clinical populations and older people and can be used as a whole questionnaire or as subscales $[19,20]$. The autonomy outdoors subscale comprises five items assessing a person's satisfaction with his/her possibilities to take part in activities outside the home: visiting relatives and friends, making trips and traveling, spending leisure time, meeting other people, and living life the way one wants to. Each item is scored from 0 (very good possibilities) to 4 (very poor possibilities). A higher sum score indicates more restrictions in autonomy in participation outdoors (range 0-20).

Self-reported physical activity was assessed using the Yale Physical Activity Survey for older adults [21]. Participants were asked how many times they had performed vigorous physical activity and leisure walking for at least 10 min during the past month and the usual duration of these sessions. Total minutes per day were calculated using the following formula [14]: (frequency*duration)/7. Finally, mean daily vigorous physical activity and leisure walking minutes were summed.

Age and sex were obtained from the Finnish National Population Register at the sampling stage. In addition, information on years of education, number of chronic conditions, depressive symptoms, and lower extremity function were collected at baseline during structured home interviews by trained interviewers and used only for descriptive purposes. Years of education, as an indicator of socioeconomic status, was self-reported. Number of chronic conditions was calculated as the sum of individual chronic conditions from a list of physician-diagnosed chronic conditions followed by an open-ended question on any other chronic diseases the participant might have [13]. Depressive symptoms were assessed with the Center for Epidemiologic Studies Depression Scale, CES-D (range
0-60, with higher scores indicating more depressive symptoms) [22]. The Short Physical Performance Battery, SPPB (range $0-12$, with higher scores indicating better lower extremity function) including balance, walking speed and chair stands were used to assess lower extremity function [23].

\section{Statistical analyses}

Baseline characteristics were compared between the selfreported walking categories using cross-tabulation with chisquare test for categorical variables and one-way ANOVA with a Bonferroni test (post hoc comparisons) for normally distributed continuous variables. Overall longitudinal changes in life-space mobility and autonomy in participation outdoors scores, and in physical activity minutes were calculated using paired samples $t$-test. Generalized Estimation Equations (GEE) linear models [24] with an unstructured working correlation matrix were used to compare changes in life-space mobility, autonomy in participation outdoors and self-reported physical activity over the followup between the self-reported walking categories. We estimated main effects (group difference) and time interaction effects (group by time). Adjusting the models for age and sex did not change the main and time interaction effects, and thus only age- and sex-adjusted models are reported. The models were adjusted only for age and sex, because the purpose was to study changes over time at the individual level in life-space mobility, autonomy in participation outdoors and self-reported physical activity related to the COVID-19 restrictions according participants' self-reported walking at baseline.

This study comprised AGNES participants who also participated in the AGNES-COVID-19 survey $(N=809)$. Age and sex were available for all participants, whereas information on self-reported walking was missing for 12 participants; hence, the final models comprised 797 participants. Missing autonomy in participation outdoors scores was imputed for follow-up participants with only one missing item $(n=14)$ using the mean of the available items. In addition, in the GEE models, multivariate imputation by chained equations was used to calculate scores for missing baseline and follow-up total scores for life-space mobility (baseline $n=4$, follow-up $n=6$ ), autonomy in participation outdoors (baseline $n=13$, follow-up $n=27$ ) and minutes for self-reported physical activity (baseline $n=14$, followup $n=16$ ). Including participants with imputed items, total scores or, minutes did not change the results based on the sensitivity analyses (data not shown). IBM SPSS version 24 for Windows (IBM Corp., Armonk, NY) was used for statistical analyses. The results were regarded as statistically significant if the $\mathrm{p}$ value was $<0.05$. 


\section{Results}

Baseline characteristics according to self-reported walking are shown in Table 1. Based on the post hoc comparisons, those reporting walking difficulty $(n=268)$ were older, less educated and had more chronic conditions, depressive symptoms, and poorer lower extremity function than those reporting intact walking $(n=396)(p<0.008$ for all). Participants with walking modifications $(n=133)$ did not differ from those with intact walking in years of education $(p=0.097)$ or number of chronic conditions $(p=0.139)$. They formed an intermediate group in their lower extremity function and depressive symptoms scores between those with intact walking and those with walking difficulty $(p<0.015$ for all) and had fewer chronic conditions than those with walking difficulty $(p<0.001)$.

Life-space mobility scores decreased on average -11.4 points (SD 21.3) in all participants during the COVID19 restrictions when compared to their scores two years before (72.6, SD 18.6 vs. 61.2, SD 24.7). Those with walking difficulty had a lower life-space mobility score at baseline (Table 1 and Fig. 1) and showed a steeper decline over time than those with intact walking. Those with walking modifications also had a lower life-space mobility score at baseline and showed a steeper decline over the follow-up than those with intact walking.

Participants were less satisfied with their possibilities to participate in activities outside their homes than two years earlier (5.1, SD 3.7 vs. 11.7, SD 5.1), as their autonomy in participation outdoors scores increased on average by 6.7 (SD 5.4) points over the follow-up. While those with walking difficulty reported poorer opportunities to participate in out-of-home activities than those with intact walking at baseline (Table 1 and Fig. 1), the decrease in autonomy in participation outdoors at follow-up was greater among those reporting intact walking at baseline. In turn, while those with walking modifications perceived worse autonomy in participation outdoors at baseline than those reporting intact walking, the change at follow-up in these two groups was similar.

Daily time spent in vigorous physical activities and in leisure walking had increased on average by 5.3 (SD 25.0) minutes among all participants at follow-up during the COVID-19 restrictions (35.3, SD 20.8 vs. 40.6, SD 27.5). At baseline, those reporting walking difficulty or use of walking modifications accumulated less daily vigorous physical activity and walking minutes than those with intact walking (Table 1 and Fig. 1). Among those with intact walking, daily vigorous physical activity and walking minutes had increased from baseline to the COVID-19 restrictions, whereas it remained more stable among those with walking difficulty and those with walking modifications.

\section{Discussion}

The present findings indicate that while life-space mobility and autonomy in participation outdoors declined, physical activity increased among community-dwelling older people between the pre-COVID baseline and the follow-up two years later during the COVID-19 restrictions. People with intact walking in 2-km distance had the most favorable baseline scores for life-space mobility, autonomy in participation outdoors and physical activity. Moreover, although their life-space mobility and autonomy in participation outdoors declined, the amount of time spent in vigorous physical activity and walking increased. In turn, those reporting

Table 1 Participants' Background Characteristics by Self-Reported Ability to Walk 2-km at Baseline ( $n=797)$

\begin{tabular}{|c|c|c|c|c|}
\hline \multirow[t]{2}{*}{ Characteristics } & \multirow{2}{*}{$\begin{array}{l}\text { Intact Walking } \\
(n=396) \\
\text { Mean (SD) }\end{array}$} & \multirow{2}{*}{$\begin{array}{l}\text { Modifications }(n=133) \\
\text { Mean (SD) }\end{array}$} & \multicolumn{2}{|c|}{ Difficulty $(n=268)$} \\
\hline & & & Mean (SD) & $P$ value \\
\hline Age, years & $77.7(3.2)$ & $78.8(3.6)$ & $79.7(3.7)$ & $<0.001^{\mathrm{a}}$ \\
\hline Education, years & $12.3(4.3)$ & $11.3(4.3)$ & $11.2(4.2)$ & $0.005^{\mathrm{a}}$ \\
\hline SPPB, score & $11.0(1.2)$ & $10.2(1.7)$ & $8.8(2.7)$ & $<0.001^{\mathrm{a}}$ \\
\hline CES-D, score & $6.6(5.9)$ & $8.5(7.2)$ & $10.7(7.7)$ & $<0.001^{\mathrm{a}}$ \\
\hline No. of chronic diseases & $2.8(1.7)$ & $3.1(1.8)$ & $4.4(2.2)$ & $<0.001^{\mathrm{a}}$ \\
\hline Life-space mobility, score & $79.8(14.9)$ & $73.5(14.9)$ & $61.4(19.7)$ & $<0.001^{\mathrm{a}}$ \\
\hline Autonomy in participation outdoors, score & $4.0(3.2)$ & $5.4(3.3)$ & $6.6(4.0)$ & $<0.001^{\mathrm{a}}$ \\
\hline Self-reported physical activity, minutes & $43.3(20.4)$ & $35.0(18.1)$ & $24.1(16.9)$ & $<0.001^{\mathrm{a}}$ \\
\hline Women, \% (n) & $52.3(207)$ & $57.1(76)$ & $68.3(183)$ & $<0.001^{b}$ \\
\hline
\end{tabular}

$S D$ Standard Deviation, $C E S$-D Center for Epidemiologic Studies Depression Scale, SPPB Short Physical Performance Battery

${ }^{a}$ Tested with one-way analysis of variance

b Tested with chi-square test 


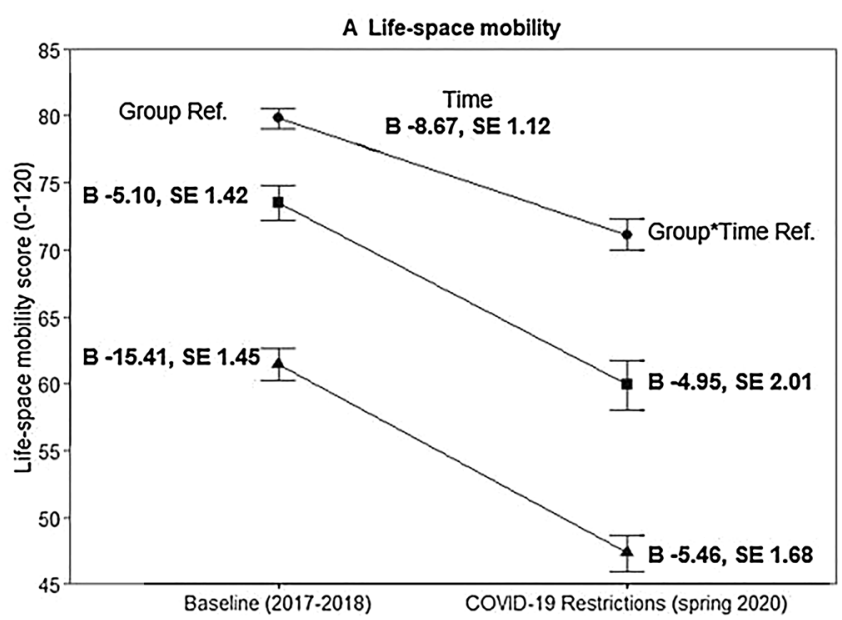

C Self-reported physical activity

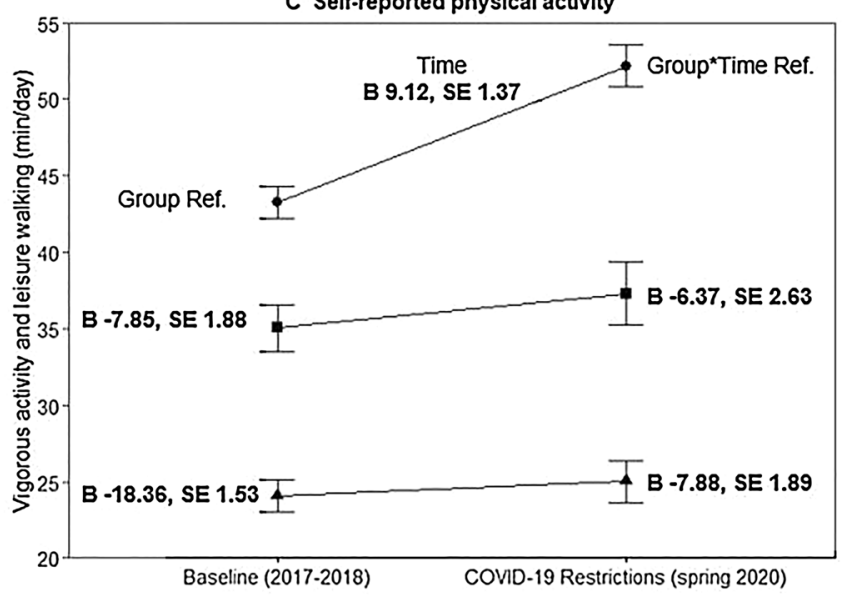

Fig. 1 Differences at baseline and in changes over time in (A) lifespace mobility (higher scores indicate greater life-space mobility), (B) autonomy in participation outdoors (higher scores indicate more restrictions in autonomy) and (C) self-reported physical activity,

walking difficulty showed a more unfavorable level of outdoor mobility at baseline and the steepest decline in lifespace mobility at follow-up during the COVID-19 restrictions. The participants with walking modifications remained in an intermediate position in all three outcome variables at both measurement points.

The decline in life-space mobility during the COVID-19 restrictions compared to two years before the pandemic was clinically meaningful [17] and notably steeper (on average 6-18 points) than in our previous study (on average 1-5 points) with a similar cohort and follow-up period [12]. Reduced life-space mobility may have a significant influence on older persons' everyday lives, as it is associated with multiple adverse health outcomes, such as increased risk for further functional decline [25], nursing home admission [26] and mortality [27]. Older people with walking difficulty and those with walking modifications showed the steepest decline in life-space mobility and were at the highest risk

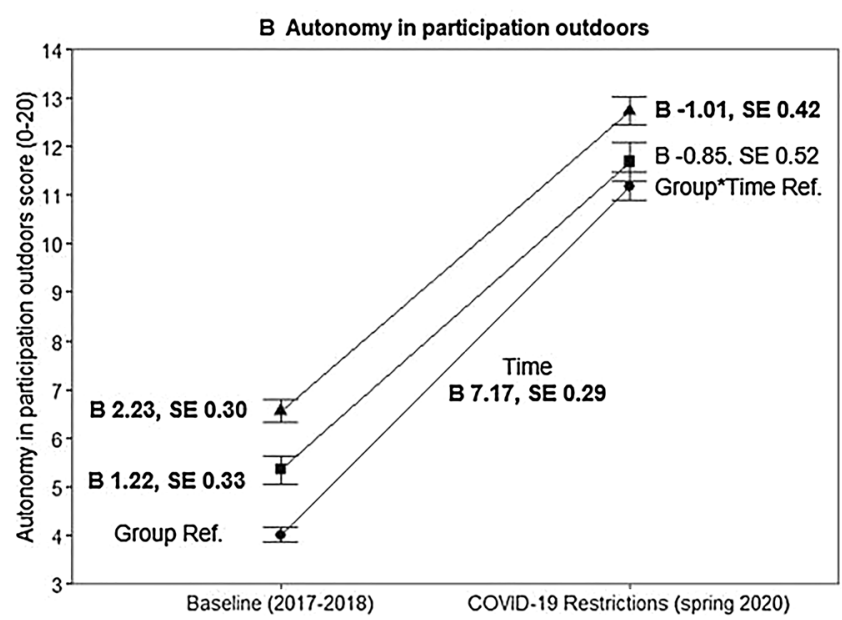

- = Intact walking ( $n=396$, ref.)

a = Walking modifications $(n=133)$

$\Delta=$ Walking difficulty $(n=268)$

vigorous activity, and leisure walking minutes (with standard error) according to self-reported walking at baseline. GEE models are adjusted for age and sex

for restricted life-space mobility (from 61 to 47 points, 74 to 60 , respectively) during the COVID-19 restrictions, meaning that they rarely moved outside of their immediate neighborhood [18]. The observed change in life-space mobility among those using walking modifications suggests that the compensatory effect of using walking modifications decreased during COVID-19 restrictions. Thus, in the present study, instead of postponing the decline in outdoor mobility [4], the use of walking modifications was an indicator of preclinical disability and a further reduction in walking activity [28]. Walking difficulty often coexists with cognitive impairments [29] and fear of moving outdoors [30], which may also compromise participation in everyday activities and accelerate the decline in life-space mobility [31]. Older people with walking difficulty may have been and may continue to be at heightened risk of becoming homebound during the COVID-19 restrictions especially if the restrictions on outdoor mobility are prolonged and 
effective interventions are not offered. Being homebound is a serious situation, as it is associated with a high mortality rate [32] and dependency in self-care [33].

Autonomy in participation outdoors indicates an individual's level of satisfaction with their opportunities to move outdoors and for instance to leave the home to visit relatives and friends as often as one wants [19]. Avoiding seeing other people was strongly recommended in Finland during the COVID-19 restrictions, and thus it is only logical that participants' perceived autonomy in participation outdoors declined (average change 6-7 points). In contrast, in our previous study, conducted during a period with no restrictions in place, perceived autonomy in participation outdoors remained almost unchanged (average change $0-1$ points) with a cohort and follow-up time comparable to those in the present study [12]. Hence, it is likely that the observed changes in participants' autonomy in participation outdoors reflect the impact of the COVID-19 restrictions rather than the impacts of a person's individual ability [19]. Our observation that people with intact walking perceived a steepest decline in their autonomy in participation outdoors compared to those perceiving walking difficulty further supports this explanation. Autonomy is an essential goal of rehabilitation as it reflects participants' own perceptions of their possibilities to live life as they want to [19] and contributes to maintaining life satisfaction [34]. Therefore, although no cut-point for a meaningful change in the autonomy in participation outdoors score has been established, the observed seven-point mean decline may have had a meaningful negative effect on the participants' lives. However, how this decline in autonomy in participation outdoors, if prolonged, affects older people's lives warrants further research.

Older people's physical activity increased in the present study, whereas in previous studies conducted in Italy and Spain it decreased during the COVID-19 restrictions [8-10]. This unexpected inconsistency between findings may be explained by the different strategies used to prevent the spread of the virus. Italy and Spain were in nationwide lockdowns and their citizens were not allowed to leave their homes without a valid reason [35, 36], whereas in Finland, no curfew was imposed at any time during spring 2020. In addition, we assessed physical activity as time spent in vigorous activity and leisure walking. In addition to exercising outdoors, vigorous activity may have included at-home activities, such as indoor cycling or strength-training. Overall, our findings suggest, in line with a previous study [37], that older people with intact walking compensated, at least partly, for their lost participation in social activities by exercising at home or walking for leisure during the COVID-19 restrictions. In contrast, the lowest levels of physical activity were observed, as in previous study [38] among older people perceiving walking difficulty. Therefore, interventions aiming to increase physical activity should especially target people perceiving walking difficulty or using walking modifications.

The study has its limitations. Owing to the COVID-19 restrictions, the follow-up data were collected using postal questionnaires. Thus, we cannot be sure who responded to the questionnaire or whether some participants misunderstood some of the questions. In addition, physical activity was self-reported, which may have led to overestimation of physical activity levels. We cannot rule out the possibility that changes in health are affecting the associations found. However, considering the greater changes in outdoor mobility in the present study compared to an earlier cohort [12] and the low rates of markedly worsened health during the follow-up, we consider that effects of the COVID-19 restrictions likely to be of greater magnitude. Overall, the effects of these limitations to the results are likely to be small.

The strengths of this study include the large populationbased sample of community-dwelling older people and the longitudinal study design with data collected prior to and during the COVID-19 restrictions. In addition, our study contributes further knowledge on the consequences of the COVID-19 restrictions: first, by assessing differences based on 2-km walking categories, second, by assessing three important aspects of older people's outdoor mobility, and third, by comparing the results over time. Previous studies, in contrast, have focused solely on changes in physical activity [8-10] or used a cross-sectional design and targeted selfselected convenience samples $[8,10]$. Finally, the present study opens the way for future research.

\section{Conclusion}

Older people with intact walking coped better with the COVID-19-related restrictions than those with walking modifications or difficulty, as they were able to compensate for suspended social activities by increasing their physical activity. In future, special attention should be paid to older people perceiving walking difficulty, as they seem to be at the highest risk for becoming homebound when environmental facilitators to outdoor mobility are removed. When comparing our findings to previous study, with a similar cohort and living environment, we noticed that the decline in life-space mobility and autonomy in participation during the first wave of COVID-19 exceeded the decline that would naturally have occurred due to the aging process over a 2-year period. As this study describes the situation in the early phase of the pandemic, further studies are needed to investigate the effects of prolonged COVID-19 restrictions on older people's outdoor mobility. In addition, studies should examine how experiencing restricted life-space mobility and autonomy in participation outdoors during the first wave of COVID-19 affects older people's subsequent 
walking ability, and whether older peoples' life-space mobility and autonomy in participation outdoors returns to preCOVID levels after the COVID-19 pandemic restrictions have been lifted.

Acknowledgements The Gerontology Research Center (GEREC) is a joint effort between the University of Jyväskylä and the University of Tampere, Finland.

Funding Open access funding provided by University of Jyväskylä (JYU). This work was supported by the European Research Council (grant number 693045 to Ta.R), the Academy of Finland (grant numbers 310526 to Ta.R, 330,185 to MR, and 321,336 and 328,818 to Ti.R), the Finnish Ministry of Education and Culture (to MR, Ta.R and $\mathrm{EP}$ ), and the University of Jyväskylä. The financial sponsors were not involved in the design, implementation, analyses or reporting of the results. In addition, the content of this manuscript does not reflect the official opinion of the European Union. Responsibility for the information and views expressed in the manuscript lies entirely with the authors.

\section{Declarations}

Conflict of interest The authors declare that they have no conflict of interest.

Statement of human and animal rights The study protocols in both data collections were performed in accordance with the ethical standards of the 1964 Helsinki declaration and its later amendments. The ethical committee of the Central Finland hospital district provided an ethical statement of the AGNES study on August 23, 2017, and May 13, 2020.

Informed consent All participants signed an informed consent prior to the start of the study.

Open Access This article is licensed under a Creative Commons Attribution 4.0 International License, which permits use, sharing, adaptation, distribution and reproduction in any medium or format, as long as you give appropriate credit to the original author(s) and the source, provide a link to the Creative Commons licence, and indicate if changes were made. The images or other third party material in this article are included in the article's Creative Commons licence, unless indicated otherwise in a credit line to the material. If material is not included in the article's Creative Commons licence and your intended use is not permitted by statutory regulation or exceeds the permitted use, you will need to obtain permission directly from the copyright holder. To view a copy of this licence, visit http://creativecommons.org/licenses/by/4.0/.

\section{References}

1. Guralnik JM, Ferrucci L, Simonsick EM, et al (1995) Lowerextremity function in persons over the age of 70 years as a predictor of subsequent disability. N Engl J Med 332:556-562. https:// doi.org/10.1056/NEJM199503023320902

2. Rantanen T (2013) Promoting mobility in older people. J Prev Med Public Health 46:S50

3. Verbrugge LM, Jette AM (1994) The disablement process. Soc Sci Med 38:1-14

4. Rantakokko M, Portegijs E, Viljanen A et al (2017) Task modifications in walking postpone decline in life-space mobility among community-dwelling older people: a 2-year follow-up study. J Gerontol A Biol Sci Med Sci 72:1252-1256. https://doi.org/10. 1093/gerona/glw348

5. Tsai L, Rantakokko M, Viljanen A, et al (2016) Associations between reasons to go outdoors and objectively-measured walking activity in various life-space areas among older people. J Aging Phys Act 24:85-91

6. Davis MG, Fox KR, Hillsdon M, et al (2011) Getting out and about in older adults: the nature of daily trips and their association with objectively assessed physical activity. Int J Behav Nutr Phys Act 8:116. https://doi.org/10.1186/1479-5868-8-116

7. Portegijs E, Tsai L, Rantanen T et al (2015) Moving through lifespace areas and objectively measured physical activity of older people. PLoS ONE 10:e135308

8. Duncan GE, Avery AR, Seto E, et al (2020) Perceived change in physical activity levels and mental health during COVID19: Findings among adult twin pairs. PLoS ONE 15:e237695. https://doi.org/10.1371/journal.pone.0237695

9. Visser M, Schaap LA, Wijnhoven HA (2020) Self-reported impact of the COVID-19 pandemic on nutrition and physical activity behaviour in dutch older adults living independently. Nutrients 12:3708. https://doi.org/10.3390/nu12123708

10. Yamada M, Kimura Y, Ishiyama D et al (2020) Effect of the COVID-19 epidemic on physical activity in community-dwelling older adults in Japan: a cross-sectional online survey. J Nutr Health Aging 24:948-950. https://doi.org/10.1007/s12603-020-1501-6

11. Rantanen T, Eronen J, Kauppinen M et al (2020) Life-space mobility and active aging as factors underlying quality of life among older people before and during COVID-19 lock-down in finland - a longitudinal study. J Gerontol A Biol Sci Med Sci 76:e60-e67. https://doi.org/10.1093/gerona/glaa274

12. Skantz H, Rantanen T, Palmberg L et al (2020) Outdoor mobility and use of adaptive or maladaptive walking modifications among older people. J Gerontol: Series A 75:806-812. https://doi.org/10. 1093/gerona/glz172

13. Rantanen T, Saajanaho M, Karavirta L et al (2018) Active agingresilience and external support as modifiers of the disablement outcome: AGNES cohort study protocol. BMC Public Health 18:565

14. Portegijs E, Karavirta L, Saajanaho M et al (2019) Assessing physical performance and physical activity in large populationbased aging studies: home-based assessments or visits to the research center? BMC Public Health 19:1570

15. Rantakokko M, Portegijs E, Viljanen A et al (2016) Mobility modification alleviates environmental influence on incident mobility difficulty among community-dwelling older people: a two-year follow-up study. PLoS ONE 11:e0154396. https://doi.org/10. 1371/journal.pone.0154396

16. Mänty M, Heinonen A, Leinonen R et al (2007) Construct and predictive validity of a self-reported measure of preclinical mobility limitation. Arch Phys Med Rehabil 88:1108-1113. https://doi. org/10.1016/j.apmr.2007.06.016

17. Baker PS, Bodner EV, Allman RM (2003) Measuring life-space mobility in community-dwelling older adults. J Am Geriatr Soc 51:1610-1614. https://doi.org/10.1046/j.1532-5415.2003.51512.x

18. Portegijs E, Iwarsson S, Rantakokko M et al (2014) Life-space mobility assessment in older people in Finland; measurement properties in winter and spring. BMC Res Notes 7:323. https:// doi.org/10.1186/1756-0500-7-323

19. Cardol M, de Haan RJ, de Jong BA et al (2001) Psychometric properties of the impact on participation and autonomy questionnaire. Arch Phys Med Rehabil 82:210-216. https://doi.org/10. 1053/apmr.2001.18218

20. Kersten P, Cardol M, George S et al (2007) Validity of the impact on participation and autonomy questionnaire: a comparison 
between two countries. Disabil Rehabil 29:1502-1509. https:// doi.org/10.1080/09638280601030066

21. Dipietro L, Caspersen CJ, Ostfeld AM et al (1993) A survey for assessing physical activity among older adults. Med Sci Sports Exerc. https://doi.org/10.1249/00005768-199305000-00016

22. Radloff LS (1977) The CES-D scale: a self-report depression scale for research in the general population. Appl Psychol Meas 1:385-401. https://doi.org/10.1177/014662167700100306

23. Guralnik JM, Simonsick EM, Ferrucci L et al (1994) A short physical performance battery assessing lower extremity function: association with self-reported disability and prediction of mortality and nursing home admission. J Gerontol 49:M85-M94

24. Liang K, Zeger SL (1986) Longitudinal data analysis using generalized linear models. Biometrika 73:13-22. https://doi.org/10. 1093/biomet/73.1.13

25. Shimada H, Ishizaki T, Kato M et al (2010) How often and how far do frail elderly people need to go outdoors to maintain functional capacity? Arch Gerontol Geriatr 50:140-146

26. Sheppard KD, Sawyer P, Ritchie CS et al (2013) Life-space mobility predicts nursing home admission over 6 years. J Aging Health 25:907-920

27. Boyle PA, Buchman AS, Barnes LL et al (2010) Association between life space and risk of mortality in advanced age. J Am Geriatr Soc 58:1925-1930

28. Weiss CO, Wolff JL, Egleston B et al (2012) Incident preclinical mobility disability (PCMD) increases future risk of new difficulty walking and reduction in walking activity. Arch Gerontol Geriatr 54:e329-e333. https://doi.org/10.1016/j.archger.2011.08.018

29. Demnitz N, Esser P, Dawes H et al (2016) A systematic review and meta-analysis of cross-sectional studies examining the relationship between mobility and cognition in healthy older adults. Gait Posture 50:164-174

30. Rantakokko M, Mänty M, Iwarsson S et al (2009) Fear of moving outdoors and development of outdoor walking difficulty in older people. J Am Geriatr Soc 57:634-640
31. Poranen-Clark T, von Bonsdorff MB, Rantakokko M et al (2018) The temporal association between executive function and lifespace mobility in old age. J Gerontol: Series A 73:835-839

32. Ankuda CK, Husain M, Bollens-Lund E et al (2021) The dynamics of being homebound over time: A prospective study of Medicare beneficiaries, 2012-2018. J Am Geriatr Soc 69:1609-1616

33. Musich S, Wang SS, Hawkins K et al (2015) Homebound older adults: Prevalence, characteristics, health care utilization and quality of care. Geriatr Nurs 36:445-450

34. Berg AI, Hassing LB, McClearn GE et al (2006) What matters for life satisfaction in the oldest-old? Aging Mental Health $10: 257-264$

35. Tobías A. (2020) Evaluation of the lockdowns for the SARSCoV-2 epidemic in Italy and Spain after one month follow up. Sci Total Environ :138539. https://doi.org/10.1016/j.scitotenv. 2020.138539.

36. Carriedo A, Cecchini JA, Fernandez-Rio J et al (2020) COVID19, psychological well-being and physical activity levels in older adults during the nationwide lockdown in Spain. Am J Geriatr Psychiatry 28:1146-1155. https://doi.org/10.1016/j.jagp.2020.08. 007

37. Portegijs E, Keskinen KE, Tuomola E et al (2021) Older adults' activity destinations before and during COVID-19 restrictions: from a variety of activities to mostly physical exercise close to home. Health Place 68:102533

38. Manns P, Ezeugwu V, Armijo-Olivo S et al (2015) Accelerometerderived pattern of sedentary and physical activity time in persons with mobility disability: national health and nutrition examination survey 2003 to 2006. J Am Geriatr Soc 63:1314-1323

Publisher's Note Springer Nature remains neutral with regard to jurisdictional claims in published maps and institutional affiliations. 